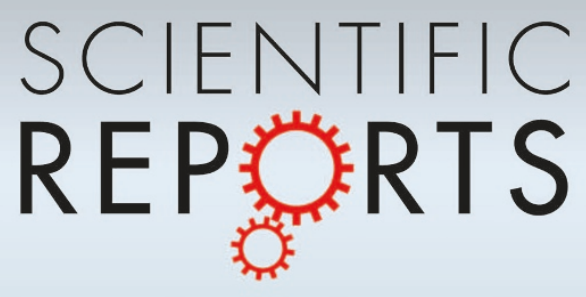

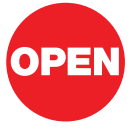

SUBJECT AREAS:

STATISTICAL PHYSICS, THERMODYNAMICS AND NONLINEAR DYNAMICS

APPLIED PHYSICS

COMPUTATIONAL BIOLOGY AND BIOINFORMATICS

POPULATION DYNAMICS

Received

9 January 2013

Accepted

1 March 2013

Published

19 March 2013

Correspondence and requests for materials should be addressed to X.L. (lix@fudan.edu.cn)

* These authors contributed equally to this work.

\section{How human location-specific contact patterns impact spatial transmission between populations?}

\author{
Lin Wang ${ }^{1 *}$, Zhen Wang ${ }^{2,3 *}$, Yan Zhang ${ }^{1} \&$ Xiang Li ${ }^{1}$
}

\begin{abstract}
'Adaptive Networks and Control Laboratory, Department of Electronic Engineering, Fudan University, Shanghai 200433, China, ${ }^{2}$ Department of Physics, Hong Kong Baptist University, Kowloon Tong, Hong Kong SAR, China, ${ }^{3}$ Center for Nonlinear Studies and the Beijing-Hong Kong-Singapore Joint Center for Nonlinear and Complex Systems (Hong Kong), Hong Kong Baptist University, Kowloon Tong, Hong Kong SAR, China.
\end{abstract}

The structured-population model has been widely used to study the spatial transmission of epidemics in human society. Many seminal works have demonstrated the impact of human mobility on the epidemic threshold, assuming that the contact pattern of individuals is mixing homogeneously. Inspired by the recent evidence of location-related factors in reality, we introduce two categories of location-specific heterogeneous human contact patterns into a phenomenological model based on the commuting and contagion processes, which significantly decrease the epidemic threshold and thus favor the outbreak of diseases. In more detail, we find that a monotonic mode presents for the variance of disease prevalence in dependence on the contact rates under the destination-driven contact scenario; while under the origin-driven scenario, enhancing the contact rate counterintuitively weakens the disease prevalence in some parametric regimes. The inclusion of heterogeneity of human contacts is expected to provide valuable support to public health implications.

T o study the epidemic spreading of viruses and diseases in human society, various mathematical models have been proposed during the past decades ${ }^{1-3}$. In particular, the network-based models have attracted a great deal of attention from diverse disciplines ${ }^{4-9}$. Plenty of works ${ }^{10-16}$ explored the transmission of etiological agent on networks, where nodes are individuals and links describe the contacts between individuals to transmit the infection.

Using the particle-network framework to mould the entire system into structured populations, we arrive at an important class of models in modern epidemiology, namely, metapopulation. In this model, individuals live in discrete subpopulations and may transfer between subpopulations via the mobility pathways (or links) ${ }^{17-26}$. The reaction-diffusion or reaction-commuting mechanism is harnessed to sketch human daily contact and mobility. More precisely, the disease prevails inside each subpopulation (with homogeneously mixed as assumed), and transmits between subpopulations through the travel of infected individuals. Applied in simulating the spatial transmission at large geographic scale, this model manifests its power in predicting the pandemic outbreak and evaluating the effectiveness of intervention strategies ${ }^{27-34}$.

Many works ${ }^{17,20,21,23,24}$ have focused on the impact of human mobility on the threshold of disease outbreak, which generalized the reaction-diffusion process to deal with heterogeneous networking populations, and assumed the mobility scheme as a Markovian memoryless migration. Recent empirical findings on human mobility ${ }^{35-39}$ report that the commuting behaviors, characterized by individual recurrent movements between connected locations, dominates the human daily transportation. Refs. 25, 26 extended the metapopulation framework by introducing the element of recurrent commuting, which assumes that individuals have the memory of their original resident subpopulation and displaced commuters who stay at the 'destination' subpopulation cannot diffuse to other places but return to their residence with a certain rate. In contrast to the random diffusion scenario, the commuting system exhibits a novel epidemic threshold as well as the phenomenon of the saturation of propagation velocity.

It is well known that the contact pattern of individuals dramatically impacts the spatiotemporal dynamics of epidemic spreading in a population ${ }^{1-3,40}$. The evolution of the epidemic process is characterized by the force of infection, which identifies the probability of acquiring infection for a susceptible individual due to his contacts with infectious ones. The force of infection is defined proportional to the density of infectious individuals, the 
infection probability via a contact between susceptible (S) and infectious (I) individuals, and the contact pattern between individuals ${ }^{1}$. Since human contact pattern is often reflected in the disease transmission rate, which is usually assumed constant $t^{1,41,42}$, in metapopulation models, the general assumption that the disease transmission rate in all subpopulations is identical indicates the fact that the individual contacts inside each subpopulation are uniform.

Within each subpopulation, the assumption of homogeneous contact (homogeneously mixing) is supported with recent empirical findings. For instance, digital equipments such as Bluetooth ${ }^{43}$, active Radio Frequency Identification (RFID) devices ${ }^{44-46}$, wireless sensors $^{47}$, and $\mathrm{WiFi}^{48,49}$, have been applied to collect the data of human close proximity contacts in various social environments, which unveils that the distribution of the number of distinct persons that each individual is in contact with per day has a small squared coefficient of variation $\left(\mathrm{CV}^{2}\right)^{45-49}$. Therefore, human contacts inside each subpopulation may possess the characteristic contact rate reflecting the number of distinct persons each individual encounters per day.

At different subpopulations, human contact pattern may present evident discrepancies. The location-specific factor has been reported as a potential driver inducing the substantial variation of disease incidence between populations in reality ${ }^{50,51}$. Generally, because of the distinction in social conditions or lifestyles, the contact rate of individuals living in an urban area is largely different from what we can expect for small-town residents. Therefore, we consider the variety of contacts between connected subpopulations to study their impact, and in this paper, we theoretically analyze this issue with the phenomenological model proposed in Ref. 52, where the recurrent commuting of individuals couples two typical subpopulations. The standard susceptible-infectious-susceptible (SIS) model is considered to reflect individuals' compartment transitions.

We first consider a general case that the individual contact features depend on the location where an individual is. This scenario illustrates the influence of social environment of the located subpopulation, and may reflect human adaptive ability ${ }^{53-55}$. Intuitively, considering the commuting flows between the urban and the suburb areas, one can expect that the contact rate for an individual commuting from the suburb to the urban area will increase, and vice versa. Therefore, we assign each individual in the located subpopulation $x$ with the same characteristic contact rate $c_{x}$, which means that on average he contacts with $c_{x}$ other individuals in the same subpopulation per unit time. We define this destination-driven mode as the type-I contact scenario.

As the commuting mobility distinguishes individuals' original residences from their destinations, we further consider another case that the contacts of individuals correlate to their own residences. This scenario may derive from the anchoring effect of human ${ }^{56,57}$, and reflects that many social factors of one's home location, e.g., economy, cultural background, inevitably affect how gregarious a person is. For instance, the schools of related disciplines of a university are usually located in the same campus (here each campus is a subpopulation), while each day students from different campuses might commute among the campuses by the school buses. Students majored in humanities or social sciences usually have a tendency to participate in social activities, and they may have a high contact rate; whereas students majored in natural sciences or medicine (particularly, graduate students) are more prone to spend their time in the laboratories or libraries, and they may own a low contact rate. Despite students from different disciplines having distinct contact rates may reside at different campuses, the commuting movements still induce their intersectional couplings in each campus per day. For simplicity, we assume that each individual from subpopulation $x$ has the characteristic contact rate $c_{x}^{\prime}$. This means that each one from $x$ will on average have contacts with $c_{x}^{\prime}$ other individuals per unit time, no matter where he locate at that time. We define this origin-driven mode as the type-II contact scenario.
In both two cases, the force of infection for a susceptible stems from all his contacts with infectious individuals per unit of time. By means of analytical arguments as well as extensive computer simulations, we demonstrate that these location-specific heterogeneous contact scenarios significantly decrease the epidemic threshold of the entire system, and thus favor the disease outbreaks in more broad parametric regimes. Under the destination-driven scenario, the variance of disease prevalence displays a monotonic mode as the contact rates change; while the results are more unexpected under the origindriven scenario: Enhancing the contact rate will weaken the disease prevalence in some parametric regimes.

\section{Results}

We first specify the mechanism of commuting mobility to transfer individuals between subpopulations. Consider two coupled subpopulations, $x, y$, each of which has a population size of the residents $N_{x}^{\star}=N_{y}^{\star}=N$. They act as the reaction places where the contagion process occurs. The model proceeds at discrete time steps, with the unit interval as one hour. Individuals leave their original resident subpopulation $x(y)$ to visit the neighboring 'destination' subpopulation $y(x)$ with a per capita diffusion rate $\sigma_{x y}\left(\sigma_{y x}\right)$ at each time step, and the displaced commuters will return to their residence $x(y)$ with a per capita return rate $\tau_{x}\left(\tau_{y}\right)$ per unit time. For simplicity, we assume the detailed balance as: $\sigma_{x y}=\sigma_{y x}=\sigma, \tau_{x}=\tau_{y}=\tau$, and Figure 1 illustrates the commuting process.

According to Refs. 26, 52, the residents of each subpopulation can be partitioned into two subgroups based on the locations where they actually are at time $t$ (see Figure 1). Define $N_{x x}(t)\left(N_{y y}(t)\right)$ the subgroup size of the individuals staying in their original resident subpopulation $x(y)$ at time $t$, and $N_{x y}(t)\left(N_{y x}(t)\right)$ the subgroup size of the individuals from subpopulation $x(y)$ presenting in the subpopulation $y(x)$ at time $t$. Therefore, the population size of the residents of each subpopulation is $N_{x}^{\star}=N_{x x}(t)+N_{x y}(t), N_{y}^{\star}=N_{y y}(t)+N_{y x}(t)$. We use the mean-field approximation to describe the commuting process with a set of rate equations governing the evolution of population dynamics $^{26,52}$, which are discussed in Supplementary Information, and we have the following equilibria:

$$
\begin{aligned}
& N_{x x}^{e q}=\frac{N_{x}^{\star}}{1+\sigma / \tau}, N_{x y}^{e q}=\frac{N_{x}^{\star}}{1+\tau / \sigma}, \\
& N_{y y}^{e q}=\frac{N_{y}^{\star}}{1+\sigma / \tau}, N_{y x}^{e q}=\frac{N_{y}^{\star}}{1+\tau / \sigma},
\end{aligned}
$$

with the condition $\sigma^{-1} \gg \tau^{-1}$, i.e., averagely, individuals will remain at their residence most of the time, which mimics that in reality only a

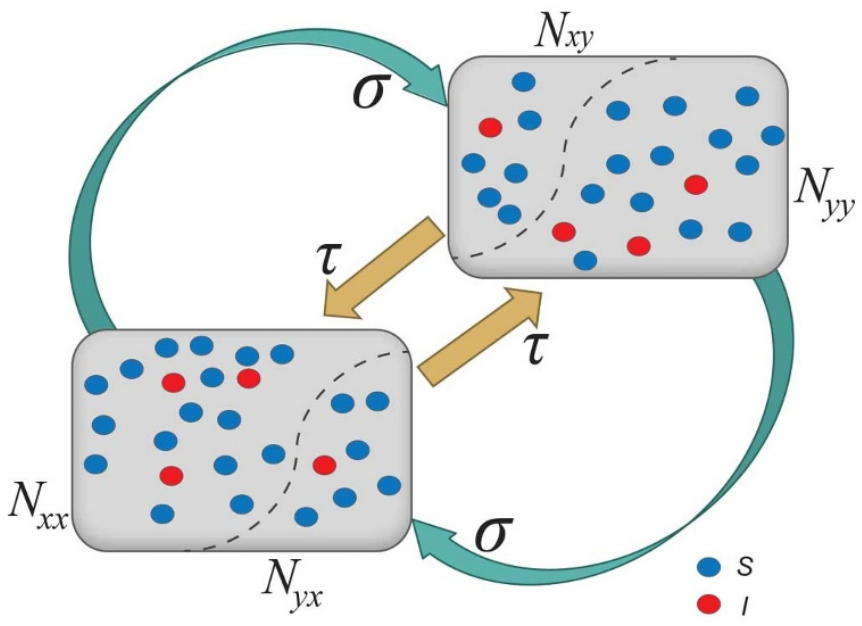

Figure $1 \mid$ Schematic illustration of the commuting process between two subpopulations $\boldsymbol{x}, \boldsymbol{y}$. The cyan and orange arrows indicate the back and forth commuting flows. 
small fraction of residents leave to travel. This condition facilitates the analysis by considering that per unit time each subpopulation $x(y)$ has an effective number of residents $N_{x y}^{e q}\left(N_{y x}^{e q}\right)$ interacting with the individuals in subgroup $N_{y y}\left(N_{x x}\right)$.

The contagion process takes place inside each subpopulation, which is composed of the infection dynamics and the contact dynamics here. For the infection dynamics, we consider the standard SIS compartment model. At time $t$, each individual falls in one of the disease compartments: susceptible or infectious. The population size, e.g., $N_{x x}(t)\left(N_{y x}(t)\right)$, can be divided into $S_{x x}(t)\left(S_{y x}(t)\right), I_{x x}(t)\left(I_{y x}(t)\right)$, which is the number of susceptible and infectious individuals in each subgroup, respectively. At each time step, if a susceptible individual contacts an infectious one, he may acquire the infection with transmission rate $\beta$. An infectious individual recovers and becomes susceptible again with rate $v$ per unit time.

We now specify the contact dynamics in more detail. In the case of the type-I (destination-driven) contact scenario, we assume that per unit time each individual staying in subpopulation $x(y)$ has the same characteristic contact rate $c_{x}\left(c_{y}\right)$. Figure S1(a) in Supporting Information illustrates this case. In the unit interval, the infection probability, $\lambda$, for a susceptible individual is determined by all his contacts with the infectious ones. Therefore, the force of infection for any susceptible in subgroup $N_{x y}$ or $N_{y y}$ at time $t$ is

$\lambda_{x y}(t)=\lambda_{y y}(t)=c_{y} \lambda_{y}(t)=\beta c_{y}\left(I_{x y}(t)+I_{y y}(t)\right) /\left(N_{x y}^{e q}+N_{y y}^{e q}\right)$,

where $\lambda_{y}(t)$ characterizes the probability that a random contact with any individual staying in subpopulation $y$ will lead to the infection at time $t$. Similarly, the force of infection for any susceptible in subgroup $N_{y x}$ or $N_{x x}$ at time $t$ is

$\lambda_{y x}(t)=\lambda_{x x}(t)=c_{x} \lambda_{x}(t)=\beta c_{x}\left(I_{y x}(t)+I_{x x}(t)\right) /\left(N_{y x}^{e q}+N_{x x}^{e q}\right)$.

With the mean-field approximation, the contagion process in each subgroup under the type-I scenario can be delineated by a set of rate equations (see Eqs.(S5)-(S8) in Supplementary Information).

We next extend to the type-II (origin-driven) contact scenario. Per unit time, an individual from subpopulation $x(y)$ has the characteristic rate of contacts, $c_{x}^{\prime}\left(c_{y}^{\prime}\right)$, which means that on average he encounters with $c_{x}^{\prime}\left(c_{y}^{\prime}\right)$ persons, no matter where he is (see Figure S1(b) for the illustration). With the mean-field approximation, the contagion process in each subgroup under the type-II scenario can also be described by the formalism of rate equations as Eqs. (S5)-(S8), except the expressions of the force of infection. The force of infection for any susceptible staying in subgroup $N_{x y}$ at time $t$ obeys the following relation

$\lambda_{x y}^{\prime}(t)=c_{x}^{\prime} \lambda_{y}^{\prime}(t)=\beta c_{x}^{\prime}\left(c_{x}^{\prime} I_{x y}(t)+c_{y}^{\prime} I_{y y}(t)\right) /\left(c_{x}^{\prime} N_{x y}^{e q}+c_{y}^{\prime} N_{y y}^{e q}\right)$,

where $\lambda_{y}^{\prime}(t)$ denotes the probability that a random contact with any individual staying in subpopulation $y$ will lead to the infection. It is worth emphasizing that to normalize the fraction of contacts of the infectious ones in each subpopulation, the denominator of Eq.(4) should be the total number of contacts of the population instead of the total number of individuals staying in the subpopulation. Similarly, the force of infection for a susceptible presenting in other subgroups at time $t$ are as follows:

$\lambda_{y y}^{\prime}(t)=c_{y}^{\prime} \lambda_{y}^{\prime}(t)=\beta c_{y}^{\prime}\left(c_{x}^{\prime} I_{x y}(t)+c_{y}^{\prime} I_{y y}(t)\right) /\left(c_{x}^{\prime} N_{x y}^{e q}+c_{y}^{\prime} N_{y y}^{e q}\right)$,

$\lambda_{y x}^{\prime}(t)=c_{y}^{\prime} \lambda_{x}^{\prime}(t)=\beta c_{y}^{\prime}\left(c_{x}^{\prime} I_{x x}(t)+c_{y}^{\prime} I_{y x}(t)\right) /\left(c_{x}^{\prime} N_{x x}^{e q}+c_{y}^{\prime} N_{y x}^{e q}\right)$,

$\lambda_{x x}^{\prime}(t)=c_{x}^{\prime} \lambda_{x}^{\prime}(t)=\beta c_{x}^{\prime}\left(c_{x}^{\prime} I_{x x}(t)+c_{y}^{\prime} I_{y x}(t)\right) /\left(c_{x}^{\prime} N_{x x}^{e q}+c_{y}^{\prime} N_{y x}^{e q}\right)$.

Besides, we consider a baseline scenario that the contact rate in each subpopulation is equal to $\langle c\rangle=\left(c_{x}+c_{y}\right) / 2$, which corresponds to the traditional situation that the contact pattern in all subpopulations is uniform. In this case, the epidemic threshold is captured by the basic reproductive number, $R_{0}^{\prime}=\beta\langle c\rangle / v$, which identifies the expected number of secondary infections produced by an infected individual during his infectious period within the entire susceptible population $^{1-3,41,58}$. If $R_{0}^{\prime}<1$, the whole system remains at the disease-free state.

Analysis. With embedding the aforementioned contact features, the epidemic threshold can be characterized by an extended version of the basic reproductive number $\mathcal{R}_{0}{ }^{2,41,58}$. We first build the next generation matrix (NGM) $T \Sigma^{-1}$ from the rate equations (S5)-(S8) at the disease-free equilibrium (DFE). Under the type-I contact scenario, the transmission matrix $T$ is defined as

$$
\begin{gathered}
T\left(I_{x x}, I_{y x}, I_{y y}, I_{x y}\right)=\left.\left(\begin{array}{ccc}
\frac{\partial T_{x x}}{\partial I_{x x}} & \cdots & \frac{\partial T_{x x}}{\partial I_{x y}} \\
\vdots & \ddots & \vdots \\
\frac{\partial T_{x y}}{\partial I_{x x}} & \cdots & \frac{\partial T_{x y}}{\partial I_{x y}}
\end{array}\right)\right|_{D F E} \\
=\left(\begin{array}{cc}
\frac{(1-\sigma) \beta c_{x} \tau}{\tau+\sigma} \frac{(1-\sigma) \beta c_{x} \tau}{\tau+\sigma} & 0 \\
\frac{(1-\tau) \beta c_{x} \sigma}{\tau+\sigma} \frac{(1-\tau) \beta c_{x} \sigma}{\tau+\sigma} & \frac{(1-\sigma) \beta c_{y} \tau}{\tau+\sigma} \frac{(1-\sigma) \beta c_{y} \tau}{\tau+\sigma} \\
0 & \frac{(1-\tau) \beta c_{y} \sigma}{\tau+\sigma} \frac{(1-\tau) \beta c_{y} \sigma}{\tau+\sigma}
\end{array}\right),
\end{gathered}
$$

while under the type-II contact scenario, the transmission matrix $T$ is

$$
\begin{aligned}
& T\left(I_{x x}, I_{y x}, I_{y y}, I_{x y}\right)=\left.\left(\begin{array}{ccc}
\frac{\partial T_{x x}}{\partial I_{x x}} & \cdots & \frac{\partial T_{x x}}{\partial I_{x y}} \\
\vdots & \ddots & \vdots \\
\frac{\partial T_{x y}}{\partial I_{x x}} & \cdots & \frac{\partial T_{x y}}{\partial I_{x y}}
\end{array}\right)\right|_{D F E}
\end{aligned}
$$

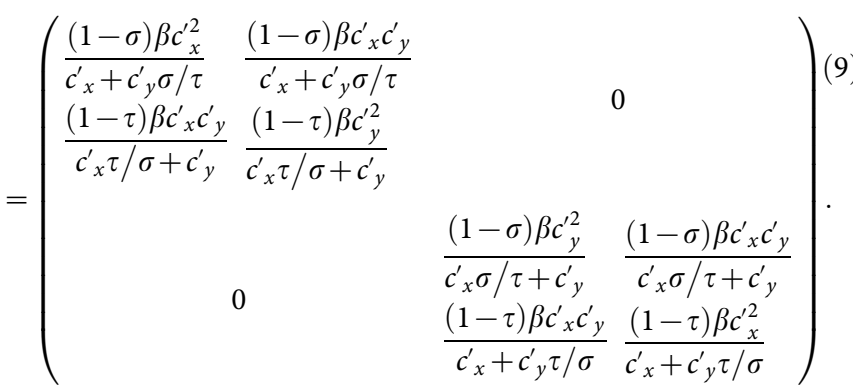

In the above two matrices, each entry $T_{i j}(i, j \in(x, y))$ represents the rate of generating new infections in subgroup $N_{i j}$. In these two scenarios, the transition matrix $\Sigma$ is

$$
\begin{aligned}
& \Sigma\left(I_{x x}, I_{y x}, I_{y y}, I_{x y}\right)=\left.\left(\begin{array}{ccc}
\frac{\partial \Sigma_{x x}}{\partial I_{x x}} & \cdots & \frac{\partial \Sigma_{x x}}{\partial I_{x y}} \\
\vdots & \ddots & \vdots \\
\frac{\partial \Sigma_{x y}}{\partial I_{x x}} & \cdots & \frac{\partial \Sigma_{x y}}{\partial I_{x y}}
\end{array}\right)\right|_{D F E} \\
& =\left(\begin{array}{cccc}
\sigma+(1-\sigma) v & 0 & 0 & -\tau \\
0 & \tau+(1-\tau) v & -\sigma & 0 \\
0 & -\tau & \sigma+(1-\sigma) v & 0 \\
-\sigma & 0 & 0 & \tau+(1-\tau) v
\end{array}\right),
\end{aligned}
$$



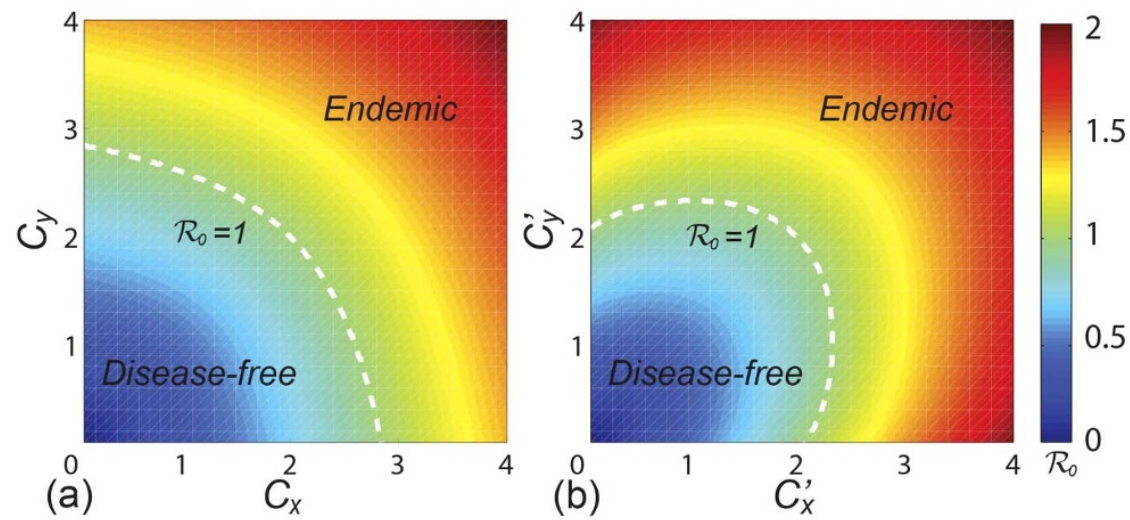

Figure $2 \mid$ The $c_{x}-c_{y}\left(c_{x}^{\prime}-c_{y}^{\prime}\right)$ phase diagrams of the global $\mathcal{R}_{\mathbf{0}}$. (a) presents the type-I contact scenario. (b) presents the type-II contact scenario. The white dashed curve in each panel illustrates the threshold of contact rates. We fix $\tau=0.125 /$ hour, $\sigma=0.042 /$ hour, $\beta=0.021 /$ hour, $v=0.042 /$ hour.

where $\Sigma_{i j}=\Sigma_{i j}^{-}-\Sigma_{i j}^{+} . \Sigma_{i j}^{+}$denotes the rate of transferring infectious individuals into subgroup $N_{i j}$, while $\Sigma_{i j}^{-}$denotes the rate of transferring infectious people out of $N_{i j}$. The recovery of infective individuals also decreases the number of infectious ones, and thus is included in $\Sigma_{i j}^{-}$. The matrices $T$ and $\Sigma$ are obtained at the disease-free equilibrium. The global version of the basic reproductive number is calculated by the spectral radius of the $\mathrm{NGM}^{41,58}$ :

$$
\mathcal{R}_{0}=\rho\left(T \Sigma^{-1}\right) \text {. }
$$

Since the explicit expression of the threshold is unavailable, we present the numerical results in the following.

Figure 2 presents the $c_{x}-c_{y}\left(c_{x}^{\prime}-c_{y}^{\prime}\right)$ phase diagrams of the global $\mathcal{R}_{0}$. As the empirical evidence tells that the commuting takes place at the time scale $\tau^{-1} \sim 1 / 3$ day $^{36}$, we choose the return rate $\tau=0.125 /$ hour. For the diffusion rate, we first set a large value $\sigma=0.042$ /hour, which implies that the time scale of diffusion is 1 day. We set other parameters of the disease as: $v=0.042 /$ hour (a short duration for the mean infectious period), and $\beta=0.021 /$ hour. Note that fixing the disease parameters as other values does not change the main results of this paper. The white dashed line in each panel indicates the parametric values corresponding to the threshold $\mathcal{R}_{0}=1$. The evident difference of the thresholds between the two heterogeneous contact scenarios shows that the type-II contact manner may induce the disease outbreak at much lower contact rates. This is due to the fact that the commuting process facilitates each subpopulation $x(y)$ to share an effective number of individuals $N_{x y}^{e q}\left(N_{y x}^{e q}\right)$ into the neighboring subpopulation $y(x)$. As the displaced commuters will closely interact with local residents, individuals with distinct contact rates have the opportunity to directly encounter in each subpopulation under the type-II scenario.

As shown in Figure 2(a), one can find a monotonic mode for the variance of global $\mathcal{R}_{0}$ under the type-I scenario, which positively correlates to the increase of contact rates $c_{x}$ and $c_{y}$. The results are more complex under the type-II scenario (see Figure 2(b)), because the values of global $\mathcal{R}_{0}$ at the upper-left and lower-right corners of the phase diagram are also very large. The variance of $\mathcal{R}_{0}$ does not show a monotonic mode when $c_{x}^{\prime}$ or $c_{y}^{\prime}$ is large $(>2.34)$ in the case of type-II scenario. For instance, when $c_{x}^{\prime}=3, \mathcal{R}_{0}$ gradually decreases until reaches the bottom, then monotonically grows with the increase of $c_{y}^{\prime}$.

We further compare the parametric regions of the endemic phases among the baseline, type-I, and type-II scenarios with the aforementioned parameters, as shown in Figure 3(a). The upper triangular light orange area corresponds to the baseline with $R_{0}^{\prime}=\beta\langle c\rangle / v>1$. The parametric space of the type-I or type-II scenario with the global $\mathcal{R}_{0}>1$ is much broader than that of the baseline case. Therefore, the inclusion of both two location-specific heterogeneous contact patterns favors the epidemic outbreak. It is also of interest to inspect the effect of a smaller diffusion rate $\sigma$ on the threshold, since reducing $\sigma$ weakens the coupling between subpopulations. Figure 3(b) compares the parametric regions pertaining to the endemic phases among the baseline, type-I, and type-II scenarios with $\sigma=0.021$ / hour, which yields the same results.

Simulations. To verify the above analysis, we use the dynamic Monte Carlo method to simulate the epidemic evolution. We focus on inspecting the parametric space of contact rates. With each set of parameters, we monitor the dynamical evolution of epidemic spreading in each subpopulation, and record the disease prevalence (the fraction of infectious individuals) in subpopulation $x(y), I_{x}(t) / N_{x}\left(I_{y}(t) / N_{y}\right)$, where $N_{x}=N_{x x}(t)+N_{y x}(t)\left(N_{y}=N_{y y}(t)\right.$ $\left.+N_{x y}(t)\right)$. Due to the presence of stochasticity, each independent Monte Carlo simulation generates a random realization of the dynamic process. We perform $10^{3}$ random Monte Carlo simulations for each set of parameters.

Initially, each subpopulation has $10^{5}$ individuals, i.e., $N_{x x}(0)=$ $N_{y y}(0)=10^{5}, N_{x y}(0)=N_{y x}(0)=0$. We fix the return rate $\tau=$ $0.125 /$ hour and the diffusion rate $\sigma=0.042$ /hour to stress the coupling effect. Figure S2 presents the time series of population evolution in each subgroup. As analyzed in Supplementary Information, the population size of each subgroup quickly reaches to the equilibrium: $N_{x x}^{e q}=N_{y y}^{e q}=7.5 \times 10^{4}, N_{x y}^{e q}=N_{y x}^{e q}=2.5 \times 10^{4}$. Then we simulate the contagion process with a variety of typical sets of contact rates, fixing the transmission parameters $\beta=0.021, v=0.042 /$ hour. The status of each individual is updated in parallel per unit time. The mobility and disease parameters are directly converted into the probabilities with the unitary time scale of one hour. The algorithm details of the simulation see the section of Methods.

We start the contagion process by introducing the seeds of infectious individuals into a given subgroup. For simplicity, the case of multiple introductions is not considered here, i.e., the seeds are only introduced into one subgroup. The contagion process begins with $I_{x x}(0)=50, S_{x x}(0)=N_{x x}^{e q}-I_{x x}(0), I_{y x}(0)=I_{y y}(0)=I_{x y}(0)=0$. By fixing the transmission parameters $\beta=0.021, v=0.042$, we mainly study the stationary prevalence (SP) $I_{x}^{e q} / N_{x}^{e q}, I_{y}^{e q} / N_{y}^{e q}$ with different parameters (SP reflects the average fraction of infectious individuals in each subpopulation at the equilibrium). To obtain each data point, we perform $10^{3}$ Monte Carlo random experiments, each of which is simulated with $10^{4}$ time steps. We define an outbreak run as the realization that the number of infectious individuals at the equilibrium is larger than 50 . The stationary prevalence is calculated by averaging over all the outbreak realizations. We first record the mean value of the number of infectious individuals in each subpopulation over the last $10 \%$ time steps of each outbreak simulation, then 

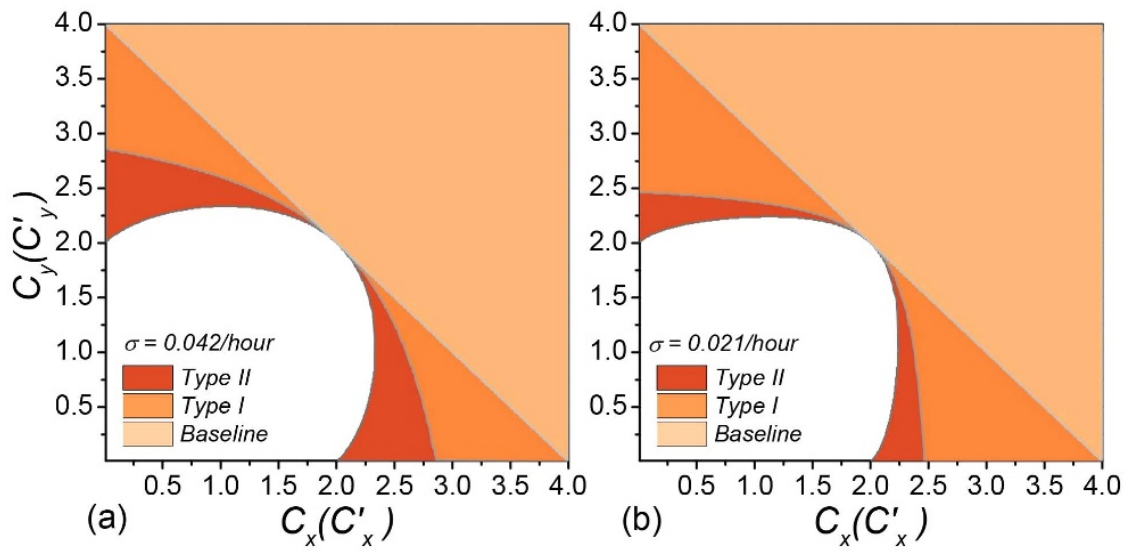

Figure 3 Comparison of the parametric space pertaining to the endemic phases among the baseline, type-I, and type-II contact scenarios. $\tau=0.125$, $v=0.042, \beta=0.021 /$ hour, and $\sigma=0.042 /$ hour in (a) and $\sigma=0.021 /$ hour in (b).

average them to obtain the stationary prevalence. If there does not exist an outbreak run, $I_{x}^{e q} / N_{x}^{e q}=I_{y}^{e q} / N_{y}^{e q}=0$.

In Figs. 4-5, we compare the phase diagrams of the stationary prevalence $I_{x}^{e q} / N_{x}^{e q}, I_{y}^{e q} / N_{y}^{e q}$ among the baseline, type-I, and typeII contact scenarios with several typical $c_{x}\left(c_{x}^{\prime}\right)$. Figures $4(\mathrm{a})-(\mathrm{b})$ show the variance of $I_{x}^{e q} / N_{x}^{e q}, I_{y}^{e q} / N_{y}^{e q}$ as $c_{y}\left(c_{y}^{\prime}\right)$ gradually increases, fixing $c_{x}=c_{x}^{\prime}=1$. The thresholds of contact rate $c_{y}\left(c_{y}^{\prime}\right)$, which separate the transition from the disease-free phase to the endemic phase, of both the type-I and type-II scenarios are much smaller than that of the baseline case, while the threshold of type-II scenario is the smallest.
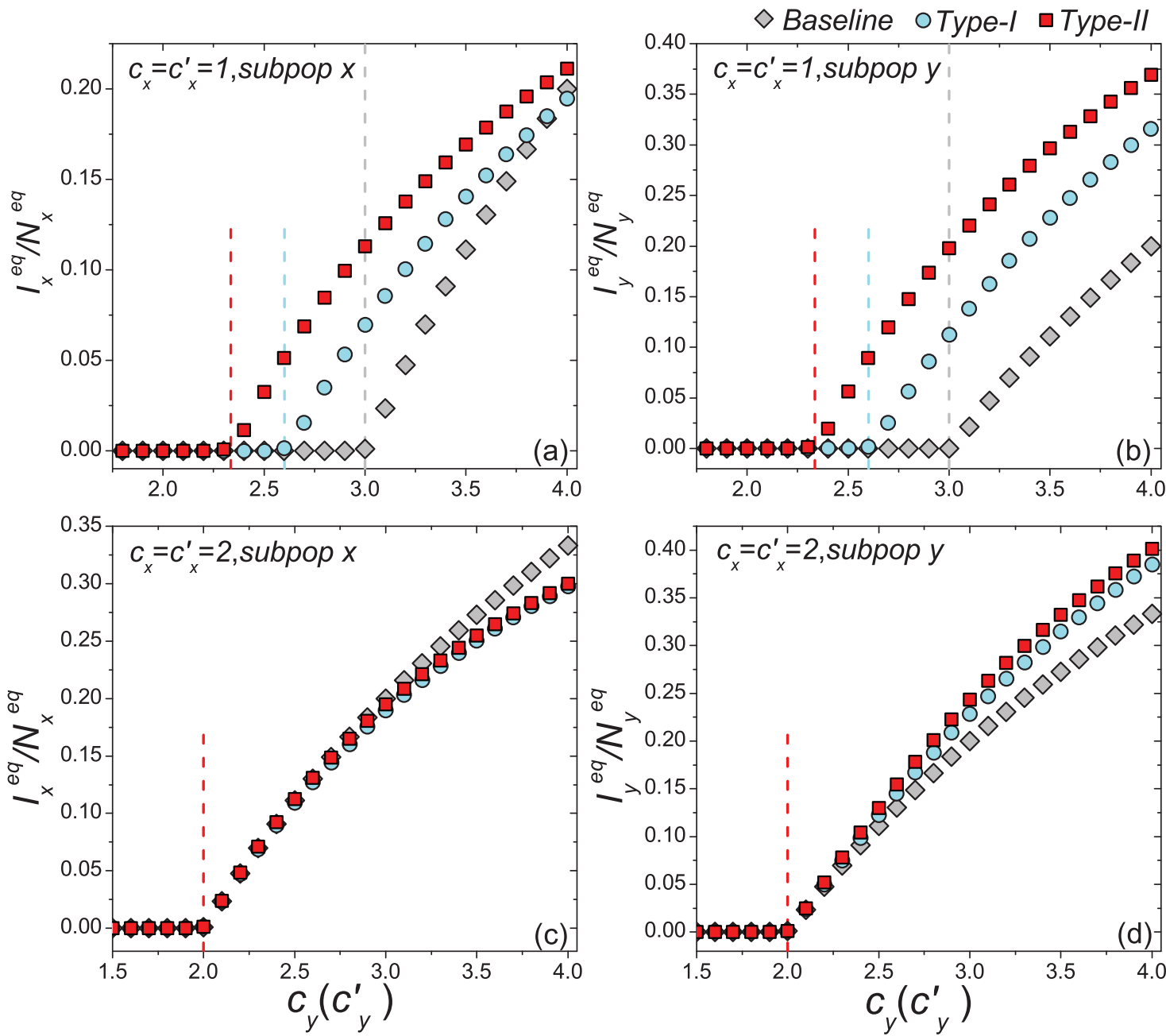

Figure $4 \mid$ Comparison of the phase diagrams of $I_{x}^{e q} / N_{x}^{e q}, I_{y}^{e q} / N_{y}^{e q}$ among the baseline, type-I, and type-II scenarios. (a)(b) show the variance of $I_{x}^{e q} / N_{x}^{e q}$ and $I_{y}^{e q} / N_{y}^{e q}$ as $c_{y}\left(c_{y}^{\prime}\right)$ gradually increases, fixing $c_{x}=c_{x}^{\prime}=1(\mathrm{c})(\mathrm{d})$ show the variance of $I_{x}^{e q} / N_{x}^{e q}$ and $I_{y}^{e q} / N_{y}^{e q}$ as $c_{y}\left(c_{y}^{\prime}\right)$ gradually increases, fixing $c_{x}=c_{x}^{\prime}=2$. The error bar is not shown since the standard deviation is much smaller (less than $10^{-1}$ of the mean value). The vertical colored dashed lines illustrate the theoretical prediction of the threshold of $c_{y}\left(c_{y}^{\prime}\right)$. 

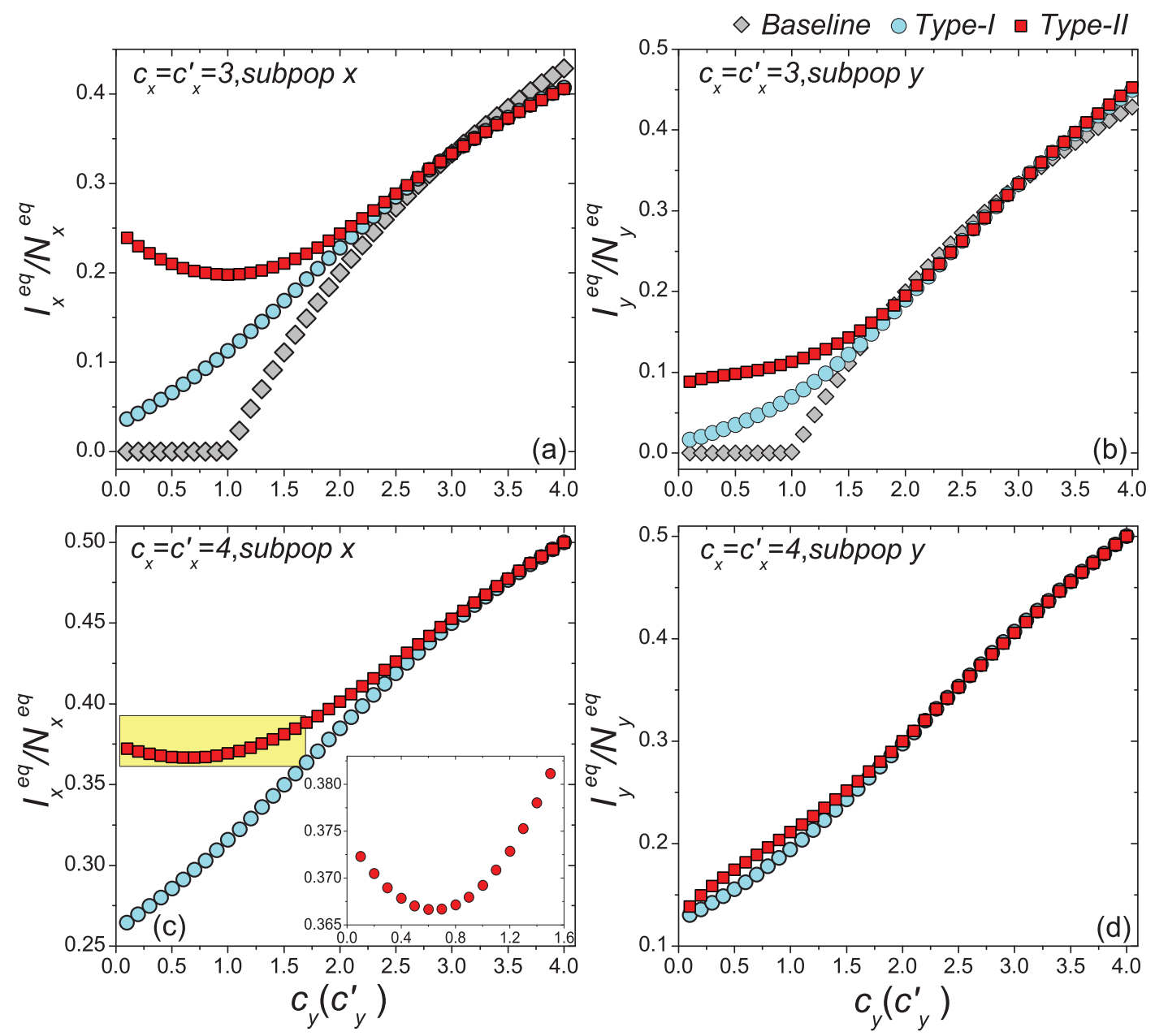

Figure 5 Comparison of the phase diagrams of $I_{x}^{e q} / N_{x}^{e q}, I_{y}^{e q} / N_{y}^{e q}$ among the baseline, type-I, and type-II contact scenarios. (a)(b) show the variance of $I_{x}^{e q} / N_{x}^{e q}$ and $I_{y}^{e q} / N_{y}^{e q}$ as $c_{y}\left(c_{y}^{\prime}\right)$ gradually increases, fixing $c_{x}=c_{x}^{\prime}=3$. (c)(d) show the variance of $I_{x}^{e q} / N_{x}^{e q}$ and $I_{y}^{e q} / N_{y}^{e q}$ as $c_{y}\left(c_{y}^{\prime}\right)$ gradually increases, fixing $c_{x}=c_{x}^{\prime}=4$. The inset in (c) shows the enlargement of the yellow area in that panel. The error bar is not shown since the standard deviation is much smaller.

We also observe the well agreement between the simulations and analysis, as illustrated by the colored dashed lines in each panel. In the endemic phase, we find a counterintuitive phenomenon that, with the same parameters, the stationary prevalence $I_{x}^{e q} / N_{x}^{e q}$, $I_{y}^{e q} / N_{y}^{e q}$ of the type-II scenario are larger than those of the type-I scenario. In subpopulation $x$, the larger $I_{x}^{e q} / N_{x}^{e q}$ of the type-II scenario might relate to the introduction of individuals with a higher contact rate. However, in subpopulation $y$, it seems a little unexpected that the type-II scenario leads to a larger $I_{y}^{e q} / N_{y}^{e q}$, because under the type-II scenario the displaced commuters from subpopulation $x$ to $y$ have a smaller contact rate when the global $\mathcal{R}_{0}>1$. In Figure 6(a), we compare the phase diagrams of global $\mathcal{R}_{0}$ between the type-I and type-II contact scenarios, fixing $c_{x}=c_{x}^{\prime}=1$. When $\mathcal{R}_{0}>1$, with the same contact rate $c_{y}=c_{y}^{\prime}$, the global $\mathcal{R}_{0}$ of the type-II scenario is evidently larger than that of the type-I scenario, which means that with the same parameters, the prevalence is more serious in the former one. Since we assume that the individual contact rate keeps unchanged in the case of type-II scenario, this outcome may be due to the fact that individuals staying in subgroup $N_{y y}$ increase their "within self-subgroup" contacts compared with those of the type-I scenario.

Figures $4(\mathrm{c})-(\mathrm{d})$ present the variance of stationary prevalence $I_{x}^{e q} / N_{x}^{e q}, I_{y}^{e q} / N_{y}^{e q}$ as $c_{y}\left(c_{y}^{\prime}\right)$ gradually increases, fixing $c_{x}=c_{x}^{\prime}=2$. In this case, the thresholds of contact rate $c_{y}\left(c_{y}^{\prime}\right)$ for the baseline, type-I, and type-II scenarios overlap at $c_{y}=c_{y}^{\prime}=2$, which is also supported by Eq.(11) as indicated by the red dashed line in each panel. In the endemic phase of $\mathcal{R}_{0}>1$, the difference of stationary prevalence between the type-I and type-II processes is largely shrunk. Figure 6(b) compares the phase diagrams of global $\mathcal{R}_{0}$ with fixing $c_{x}=c_{x}^{\prime}=2$. In the case of a large $c_{y}\left(c_{y}^{\prime}\right)$, the difference of $\mathcal{R}_{0}$ becomes smaller than that of the former situation $c_{x}=c_{x}^{\prime}=1$.

When we further increase $c_{x}\left(c_{x}^{\prime}\right)$, e.g., $c_{x}=c_{x}^{\prime}=3$ or $c_{x}=c_{x}^{\prime}=4$, the outcomes are more complex. Figures $5(\mathrm{a})-(\mathrm{b})$ show the variance of $I_{x}^{e q} / N_{x}^{e q}, I_{y}^{e q} / N_{y}^{e q}$ as $c_{y}\left(c_{y}^{\prime}\right)$ gradually increases, fixing $c_{x}=c_{x}^{\prime}=3$. In the baseline case, both the simulations and analysis agree that the threshold of contact rate decreases to $c_{y}=1$. The heterogeneous contact scenarios yield that the threshold of $c_{y}\left(c_{y}^{\prime}\right)$ vanishes. Figures 5(c)-(d) present the variance of $I_{x}^{e q} / N_{x}^{e q}, I_{y}^{e q} / N_{y}^{e q}$ in dependence on $c_{y}\left(c_{y}^{\prime}\right)$, fixing $c_{x}=c_{x}^{\prime}=4$. Since the threshold of contact rate of the baseline also vanishes, the baseline results are not included in Figures $5(\mathrm{c})(\mathrm{d})$. The analytical results of global $\mathcal{R}_{0}$ (see Figures $6(\mathrm{c})(\mathrm{d}))$ indicate that the disease-free phase is eliminated, no matter how small $c_{y}\left(c_{y}^{\prime}\right)$ is.

Moreover, under the type-I process, both $I_{x}^{e q} / N_{x}^{e q}$ and $I_{y}^{e q} / N_{y}^{e q}$ monotonically increase with the augment of $c_{y}$, as shown in Figure 5. Under the type-II process, it is evident that $I_{y}^{e q} / N_{y}^{e q}$ also monotonically increases with the growth of $c_{y}^{\prime}$, while $I_{x}^{e q} / N_{x}^{e q}$ first gradually decreases, after reaching the bottom it begins to 

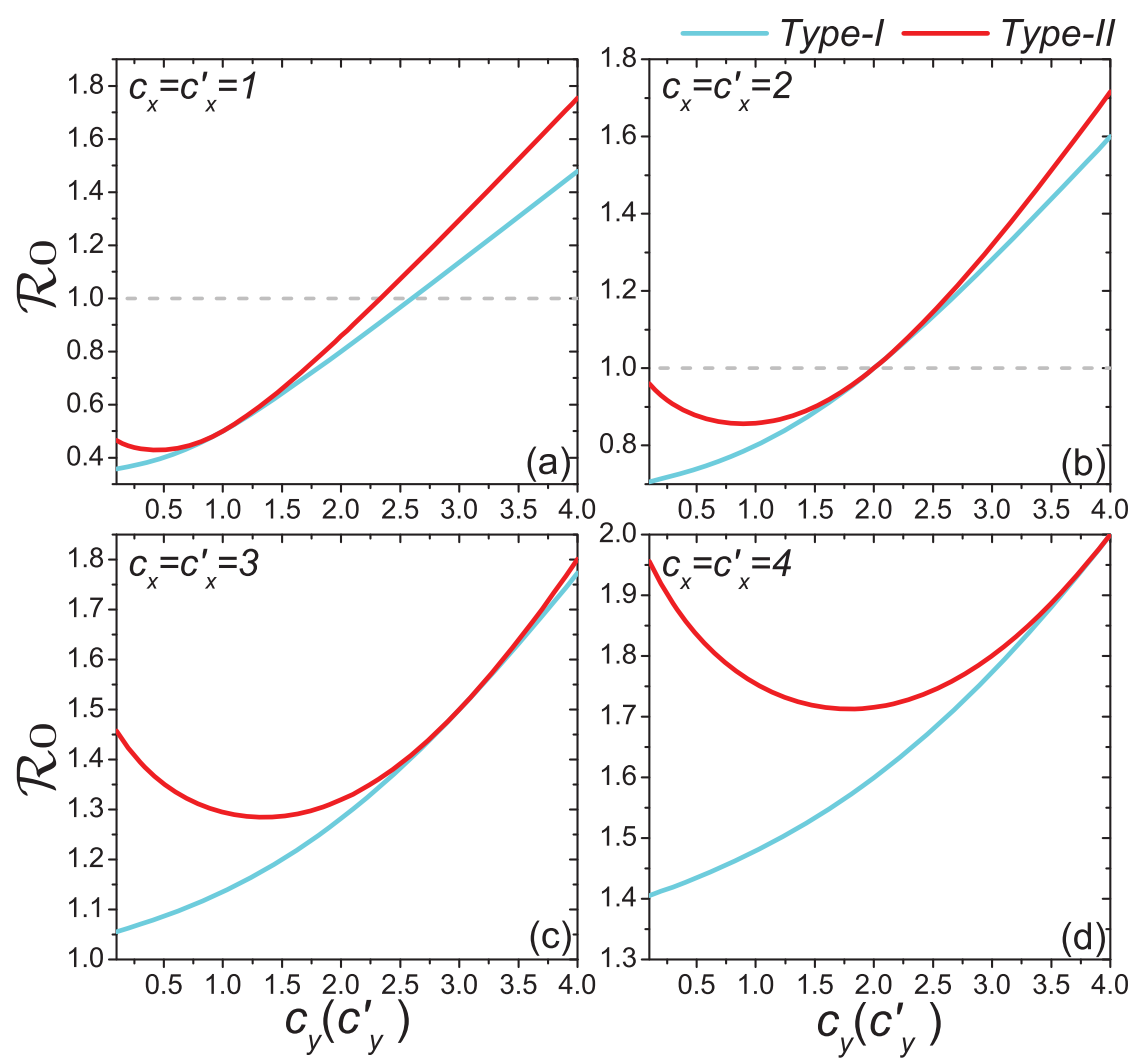

Figure 6 | Comparison of the phase diagrams of global $\mathcal{R}_{0}$ between the type-I and type-II contact scenarios. Each panel shows the variance of global $\mathcal{R}_{0}$ as $c_{y}\left(c_{y}^{\prime}\right)$ gradually increases, with $c_{x}=c_{x}^{\prime}=1$ in (a), $c_{x}=c_{x}^{\prime}=2$ in (b), $c_{x}=c_{x}^{\prime}=3$ in (c), $c_{x}=c_{x}^{\prime}=4$ in (d). The horizontal gray dashed lines in the upper two panels illustrate the threshold value $\mathcal{R}_{0}=1$.

monotonically grow with the increase of $c_{y}^{\prime}$. The variance of global $\mathcal{R}_{0}$ under the type-II scenario also experiences a valley as $c_{y}^{\prime}$ gradually increases (see Figure 6). To explain this untrivial phenomenon, we observe the stationary prevalence of each subgroup in detail. As an example, we present the results of $c_{x}^{\prime}=3$ in Figure 7. It is clear that $I_{y y}^{e q} / N_{y y}^{e q}$ and $I_{y x}^{e q} / N_{y x}^{e q}$ monotonically increase with the increment of $c_{y}^{\prime}$, whereas the variances of $I_{x x}^{e q} / N_{x x}^{e q}$ and $I_{x y}^{e q} / N_{x y}^{e q}$ are nonmonotonic. In subpopulation $x$, the probability of an individual of subgroup $N_{x x}$ to involve in a contact is $c_{x}^{\prime} /\left(c_{x}^{\prime} N_{x x}+c_{y}^{\prime} N_{y x}\right)$, while the probability of an individual of subgroup $N_{y x}$ to involve in a contact is $c_{y}^{\prime} /\left(c_{x}^{\prime} N_{x x}+c_{y}^{\prime} N_{y x}\right)$. With the increase of $c_{y}^{\prime}$, the individuals in subgroup $N_{x x}$ will reduce their 'within self-subgroup' contacts. As $c_{y}^{\prime}$ initially grows from 0.1 , the net loss of infectious individuals in subgroup $N_{x x}$ suppresses the gain obtained from subgroup $N_{y x}$, because $N_{x x}^{e q} \gg N_{y x}^{e q}$. In this stage, the presence of commuters from subpopulation $y$ mitigates the epidemic situation in subpopulation $x$, and their influence will be enhanced with the increment of $c_{y}^{\prime}$. Thus one can observe the counterintuitive drop of $I_{x}^{e q} / N_{x}^{e q}$. In subpopulation $y$, with the increase of $c_{y}^{\prime}$, the net increase of infectious individuals in subgroup $N_{y y}$ can compensate the loss of subgroup $N_{x y}$, because $N_{y y}^{e q} \gg N_{x y}^{e q}$. Therefore, $I_{y}^{e q} / N_{y}^{e q}$ keeps on increasing as shown in Figure 5(b). As subpopulations $x, y$ are coupled together, increasing $c_{y}^{\prime}$ will eventually cease the decline of $I_{x}^{e q} / N_{x}^{e q}$. After hitting the bottom, $I_{x}^{e q} / N_{x}^{e q}$ positively correlates with the increase of $c_{y}^{\prime}$.
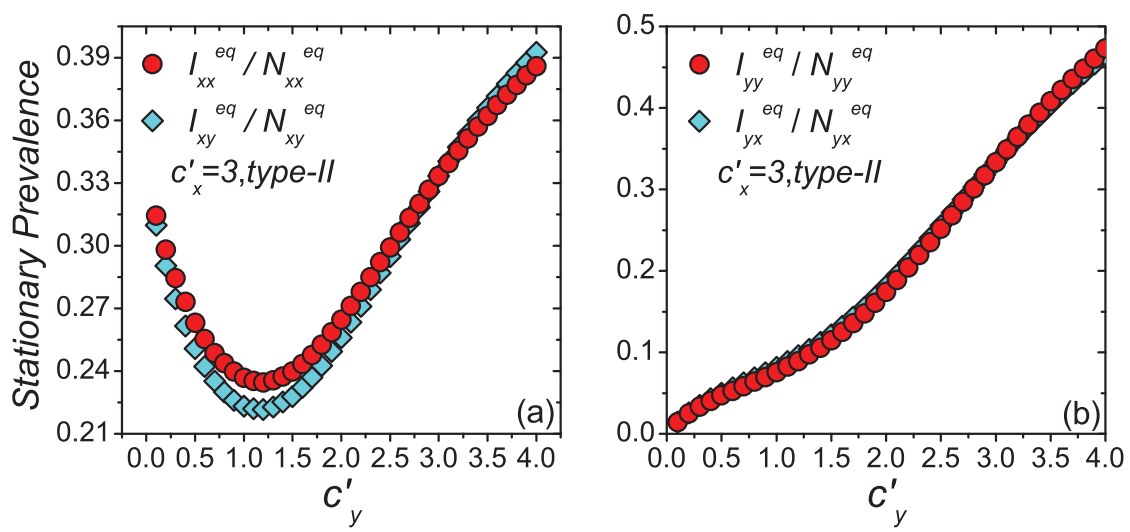

Figure $7 \mid$ Variance of the stationary prevalence of infectious individuals in subgroups $N_{x x}, N_{x y}(a), N_{y y}, N_{y x}(b)$ as $c_{y}\left(c_{y}^{\prime}\right)$ gradually increases, under the type-II scenario. We fix $c_{x}^{\prime}=3$. 

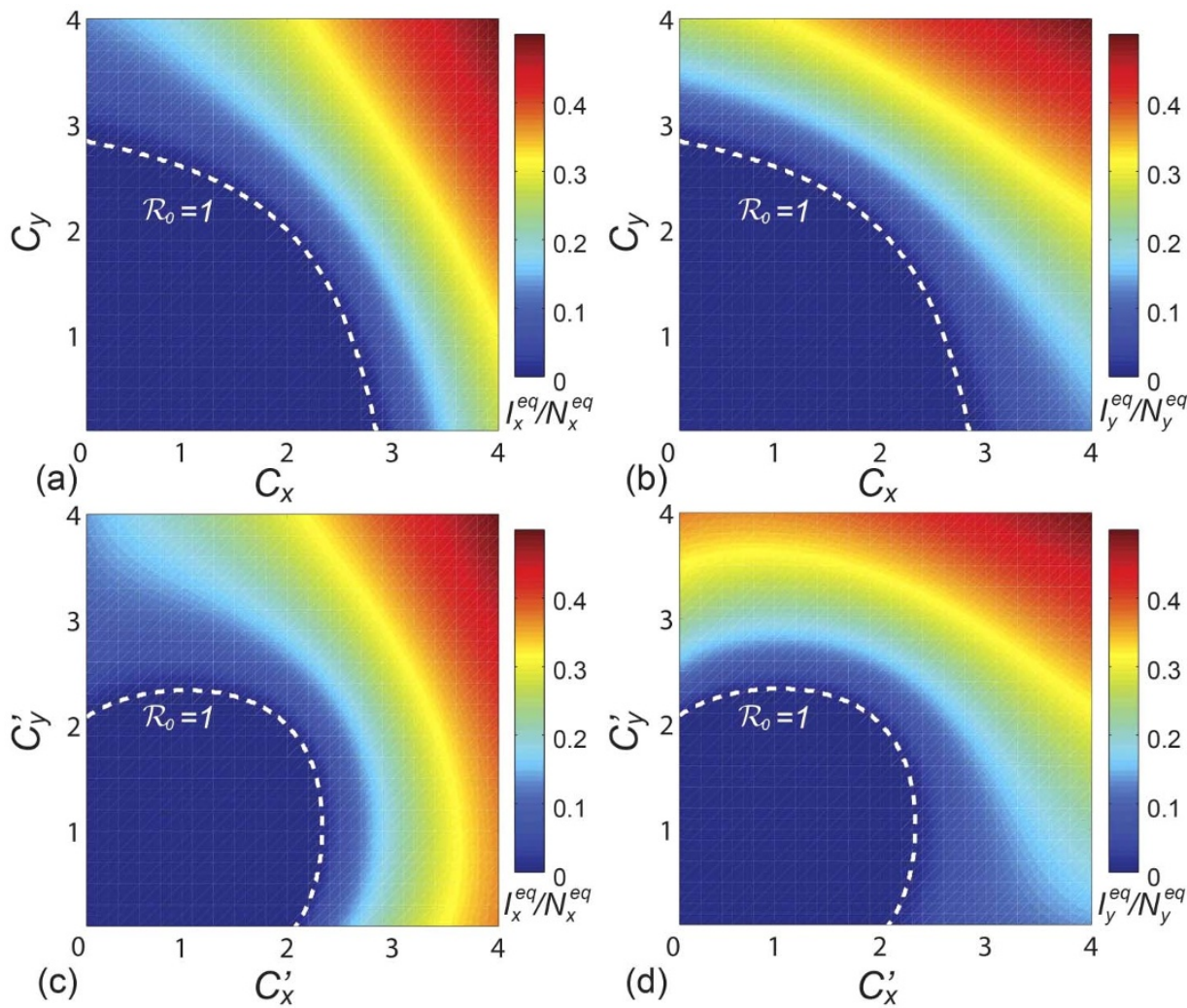

Figure $8 \mid$ The $c_{x}-c_{y}\left(c_{x}^{\prime}-c_{y}^{\prime}\right)$ phase diagrams of $I_{x}^{e q} / N_{x}^{e q}, I_{y}^{e q} / N_{y}^{e q}$. (a)(b) show the type-I scenario. (c)(d) show the type-II scenario. The white dashed curve in each panel illustrates the threshold of contact rates leading to the global $\mathcal{R}_{0}=1$.

As a brief summary, we present the $c_{x}-c_{y}\left(c_{x}^{\prime}-c_{y}^{\prime}\right)$ phase diagrams of the stationary prevalence $I_{x}^{e q} / N_{x}^{e q}$ and $I_{y}^{e q} / N_{y}^{e q}$ under the type-I and type-II scenarios, and give a holistic view about the impact of different contact patterns on the spatial transmission between populations. Figures $8(\mathrm{a})-(\mathrm{b})$ plot the $c_{x}-c_{y}$ phase diagrams of $I_{x}^{e q} / N_{x}^{e q}$ and $I_{y}^{e q} / N_{y}^{e q}$ under the type-I scenario, respectively. One can clearly observe the monotonic increase of $I_{x}^{e q} / N_{x}^{e q}$ and $I_{y}^{e q} / N_{y}^{e q}$ with the increment of $c_{x}$ and $c_{y}$. The white dashed line in each panel illustrates the threshold of contact rates leading to the global $\mathcal{R}_{0}=1$ (calculated by the NGM method), which successfully separates the endemic phase from the disease-free phase. Similarly, Figures 8(c)(d) show the $c_{x}^{\prime}-c_{y}^{\prime}$ phase diagrams of $I_{x}^{e q} / N_{x}^{e q}$ and $I_{y}^{e q} / N_{y}^{e q}$ under the type-II scenario, respectively. The parametric space corresponding to the endemic phase under the type-II scenario is broader than that of the type-I scenario. When $c_{x}^{\prime}$ or $c_{y}^{\prime}>2.34$, the stationary prevalence $I_{x}^{e q} / N_{x}^{e q}$ and $I_{y}^{e q} / N_{y}^{e q}$ does not always monotonically increase with the augment of $c_{x}^{\prime}, c_{y}^{\prime}$. In this case, the increment of contact rates will reduce the disease prevalence $I_{x}^{e q} / N_{x}^{e q}$ or $I_{y}^{e q} / N_{y}^{e q}$ in some parametric regimes.

\section{Discussion}

With the evidence of location-related factors in reality, we have introduced two categories of location-specific contact patterns in a phenomenological structured populations model based on the commuting and contagion processes. Through theoretical analysis and extensive computational simulations, we have shown that these heterogeneous contact scenarios favor the disease outbreaks and evidently decrease the epidemic threshold of the entire system. This finding is robust against the changing of system parameters. More specifically, under the destination-driven scenario, the variance of disease prevalence exhibits the monotonic dependence of contact rates, while under the origin-driven scenario the increment of contact rates unexpectedly reduces the disease prevalence in some parametric regimes. Compared to the traditional framework, where the contact pattern in all subpopulations is uniform, the models presented in this paper demonstrate that the inclusion of these extra factors raises the risk of infection, mainly due to the presence of couplings between populations. Therefore, the interventions to limit the mobility flows, e.g., entry screening, and travel restriction, may benefit to control the disease invasion. To better understand the impact of human contact behaviors on the spatial transmission of infectious disease, more efforts are deserved to deal with more heterogeneous networking populations in further studies.

\section{Methods}

Algorithm details. The contagion and commuting processes at each time step proceed as follows:

(i) Contagion process. For the type-I scenario, the probability of a susceptible staying in subpopulation $x$ to acquire the infection by each of his contacts at time $t$ is $\lambda_{x}(t)=\beta\left(I_{x x}(t)+I_{y x}(t)\right) /\left(N_{x x}(t)+N_{y x}(t)\right)$. Therefore, the force of infection for each susceptible staying in subgroup $N_{x x}$ or $N_{y x}$ is $\lambda_{x x}(t)=\lambda_{y x}(t)=c_{x} \lambda_{x}(t)$. Similarly, the force of infection for each susceptible staying in subgroup $N_{y y}$ or $N_{x y}$ at time $t$ is $\lambda_{y y}(t)$ $=\lambda_{x y}(t)=c_{y} \lambda_{y}(t)$. At this time step, the number of new infections in subgroup $N_{x y}$ is generated from a binomial distribution with probability $\lambda_{x y}(t)$ and the number of trials $S_{x y}(t)$. The number of new infections in other subgroups is generated in the same way. At the same time, any infectious individual recovers and becomes susceptible again with rate $v$. The number of recovered individuals in each subgroup is generated from a binomial distribution with probability $v$ and the number of trials defined by the number of infectious individuals in that subgroup.

For the type-II scenario, each resident of subpopulation $x(y)$ contacts with $c_{x}^{\prime}\left(c_{y}^{\prime}\right)$ other individuals in his current location per unit time. At time $t$, the probability of a susceptible presenting in subpopulation $y$ to acquire the infection by each of his

contacts is $\lambda_{y}^{\prime}(t)=\beta\left(c_{x}^{\prime} I_{x y}(t)+c_{y}^{\prime} I_{y y}(t)\right) /\left(c_{x}^{\prime} N_{x y}(t)+c_{y}^{\prime} N_{y y}(t)\right)$. Thus the force of infection of each susceptible in subgroup $N_{x y}$ is $\lambda_{x y}^{\prime}(t)=c_{x}^{\prime} \lambda_{y}^{\prime}(t)$, while the force of infection of each susceptible in subgroup $N_{y y}$ is $\lambda_{y y}^{\prime}(t)=c_{y}^{\prime} \lambda_{y}^{\prime}(t)$. The forces of infection of susceptible individuals in other subgroups are obtained similarly. At this time step, the number of new infections in subgroup $N_{x y}$ is generated from a binomial distribution with probability $\lambda_{x y}^{\prime}(t)$ and the number of trials $S_{x y}(t)$. The number of new infections in other subgroups is generated in the same way. At each time step, the 
number of recovered individuals in each subgroup is generated from a binomial distribution with probability $v$ and the number of trials defined by the number of infectious individuals in that subgroup.

(ii) Commuting process. At each time step, after all individuals update the contagion dynamics, the simulation moves to their commuting process. The number of susceptible travelers departing from their resident subpopulation $x(y)$ per unit time is generated from a binomial distribution with probability $\sigma$ and the number of trials $S_{x x}(t)\left(S_{y y}(t)\right)$. The number of susceptible commuters returning to their residence $x(y)$ per unit time is also generated from a binomial distribution with probability $\tau$ and the number of trails $S_{x y}(t)\left(S_{y x}(t)\right)$. The number of infectious individuals leaving from or returning to their residence is acquired in the same way.

1. Anderson, R. M. \& May, R. M. Infectious Diseases of Humans: Dynamics and Control (Oxford University Press, Oxford, UK, 1991).

2. Diekmann, O. \& Heesterbeek, J. A. P. Mathematical Epidemiology of Infectious Diseases: Model Building, Analysis and Interpretation (John Wiley \& Sons, New York, 2000).

3. Keeling, M. J. \& Rohani, P. Modeling Infectious Diseases in Humans and Animals (Princeton University Press, Princeton and Oxford, 2008).

4. Boccaletti, S., Latora, V., Moreno, Y., Chavez, M. \& Hwang, D. U. Complex networks: Structure and dynamics. Phys. Rep. 424, 175-308 (2006).

5. Newman, M. E. J. Networks: An Introduction (Oxford University Press, New York, 2010).

6. Perc, M. \& Szolnoki, A. Coevolutionary games - A mini review. BioSystems 99, 109-125 (2010).

7. Csermely, P. Weak Links: The Universal Key to the Stability of Networks and Complex Systems (Springer-Verlag, Berlin, Heidelberg, 2006).

8. Vespignani, A. Modelling dynamical processes in complex socio-technica systems. Nat. Phys. 8, 32-39 (2012).

9. Perc, M., Gómez-Gardeñnes, J., Szolnoki, A., Floría, L. M. \& Moreno, Y. Evolutionary dynamics of group interactions on structured populations: A review. J. R. Soc. Interface 10, 20120997 (2013)

10. Meyers, L. A., Pourbohloul, B., Newman, M. E. J., Skowronski, D. M. \& Brunham, R. C. Network theory and SARS: Predicting outbreak diversity. J. Theor. Biol. 232, 71-81 (2005).

11. Li, X. \& Wang, X. F. Controlling the spreading in small-world evolving networks: Stability, oscillation, and topology. IEEE Trans. Automat. Control 51, 534-540 (2006).

12. Zhou, T., Liu, J. G., Bai, W. J., Chen, G. R. \& Wang, B.-H. Behaviors of susceptibleinfected epidemics on scale-free networks with identical infectivity. Phys. Rev. E 74, 056109 (2006).

13. Gómez-Gardeñes, J., Latora, V., Moreno, Y. \& Profumo, E. Spreading of sexually transmitted diseases in heterosexual populations. Proc. Natl. Acad. Sci. USA 105, 1399-1404 (2008).

14. Meloni, S., Arenas, A. \& Moreno, Y. Traffic-driven epidemic spreading in finitesize scale-free networks. Proc. Natl. Acad. Sci. USA 106, 16897-16902 (2009).

15. Parshani, R., Carmi, S. \& Havlin, S. Epidemic threshold for the susceptibleinfectious-susceptible model on random networks. Phys. Rev. Lett. 104, 258701 (2010).

16. Castellano, C. \& Pastor-Satorras, R. Thresholds for epidemic spreading in networks. Phys. Rev. Lett. 105, 218701 (2010).

17. Rvachev, L. A. \& Longini Jr, I. M. A mathematical model for the global spread of influenza. Math. Biosci. 75, 3-22 (1985).

18. Watts, D., Muhamad, R., Medina, D. C. \& Dodds, P. S. Multiscale, resurgent epidemics in a hierarchical metapopulation model. Proc. Natl. Acad. Sci. USA 102, 11157-11162 (2005)

19. Viboud, C. et al. Synchrony, waves, and spatial hierarchies in the spread of influenza. Science 312, 447-451 (2006).

20. Colizza, V., Pastor-Satorras, R. \& Vespignani, A. Reaction-diffusion processes and metapopulation models in heterogeneous networks. Nat. Phys. 3, 276-282 (2007).

21. Tang, M., Liu, Z. H. \& Li, B. W. Epidemic spreading by objective traveling. Europhys. Lett. 87, 18005 (2009).

22. Wang, L., Li, X., Zhang, Y. Q., Zhang, Y. \& Zhang, K. Evolution of scaling emergence in large-scale spatial epidemic spreading. PLoS One 6, e21197 (2011).

23. Meloni, S. et al. Modeling human mobility responses to the large-scale spreading of infectious diseases. Sci. Rep. 1, 62 (2011)

24. Wang, B., Cao, L., Suzuki, H. \& Aihara, K. Safety-information-driven human mobility patterns with metapopulation epidemic dynamics. Sci. Rep. 2, 887 (2012).

25. Belik, V., Geisel, T. \& Brockmann, D. Natural human mobility patterns and spatial spread of infectious diseases. Phys. Rev. X 1, 011001 (2011).

26. Balcan, D. \& Vespignani, A. Invasion threshold in structured populations with recurrent mobility patterns. J. Theor. Biol. 293, 87-100 (2012).

27. Hufnagel, L., Brockmann, D. \& Geisel, T. Forecast and control of epidemics in a globalized world. Proc. Natl. Acad. Sci. USA 101, 15124-15129 (2004).

28. Hollingsworth, T. D., Ferguson, N. M. \& Anderson, R. M. Will travel restrictions control the international spread of pandemic influenza? Nat. Med. 12, 497-499 (2006)

29. Epstein, J. M. et al. Controlling pandemic flu: The value of international air travel restrictions. PLoS One 2, e401 (2007).
30. Cooper, B. S., Pitman, R. J., Edmunds, W. J. \& Gay, N. J. Delaying the international spread of pandemic influenza. PLoS Med. 3, e212 (2006).

31. Khan, K. et al. Spread of a novel influenza A (H1N1) virus via global airline transportation. N. Engl. J. Med. 361, 212-214 (2009).

32. Kenah, E., Chao, D. L., Matrajt, L., Elizabeth Halloran, M. \& Longini Jr., I. M. The global transmission and control of influenza. PLoS One 6, e19515 (2011).

33. Bajardi, P. et al. Human mobility networks, travel restrictions, and the global spread of 2009 H1N1 pandemic. PLoS One 6, e16591 (2011).

34. Wang, L., Zhang, Y., Huang, T. Y. \& Li, X. Estimating the value of containment strategies in delaying the arrival time of an influenza pandemic: A case study of travel restriction and patient isolation. Phys. Rev. E 86, 032901 (2012).

35. González, M. C., Hidalgo, C. A. \& Barabási, A. L. Understanding individual human mobility patterns. Nature 453, 779-782 (2008).

36. Balcan, D. et al. Multiscale mobility networks and the spatial spreading of infectious diseases. Proc. Natl. Acad. Sci. USA 106, $21484-21489$ (2009).

37. Song, C. M., Qu, Z. H., Blumm, N. \& Barabási, A.-L. Limits of predictability in human mobility. Science 327, 1018-1021 (2010)

38. Keeling, M. J., Danon, L., Vernon, M. C. \& House, T. A. Individual identity and movement networks for disease metapopulations. Proc. Natl. Acad. Sci. USA 107, 8866-8870 (2010).

39. Simini, F., González, M. C., Maritan, A. \& Barabási, A. L. A universal model for mobility and migration patterns. Nature 484, 96-100 (2012).

40. Mossong, J. et al. Social contacts and mixing patterns relevant to the spread of infectious diseases. PLoS Med. 5, e74 (2008).

41. Cao, L., Li, X., Wang, B. \& Aihara, K. Rendezvous effects in the diffusion process on bipartite metapopulation networks. Phys. Rev. E 84, 041936 (2011).

42. Fumanelli, L. et al. Inferring the structure of social contacts from demographic data in the analysis of infectious diseases spread. Plos Comput. Biol. 8, e1002673 (2012).

43. Eagle, N., Pentland, A. \& Lazer, D. Inferring friendship network structure by using mobile phone data. Proc. Natl. Acad. Sci. USA 106, 15274-15278 (2009).

44. Cattuto, C. et al. Dynamics of person-to-person interactions from distributed RFID sensor networks. PLoS One 5, e11596 (2010).

45. Isella, L. et al. What's in a crowd? Analysis of face-to-face behavioral networks. J. Theor. Biol. 271, 166-180 (2011).

46. Takaguchi, T., Nakamura, M., Sato, N., Yano, K. \& Masuda, N. Predictability of conversation partners. Phys. Rev. X 1, 011008 (2011)

47. Salathé, M. et al. A high-resolution human contact network for infectious disease transmission. Proc. Natl. Acad. Sci. USA 107, 22020-22025 (2010).

48. Zhang, Y., Wang, L., Zhang, Y. Q. \& Li, X. Towards a temporal network analysis of interactive WiFi users. Europhys. Lett. 98, 68002 (2012).

49. Zhang, Y. Q. \& Li, X. Characterizing large-scale population's indoor spatiotemporal interactive behaviors. Proc. ACM SIGKDD Int. Workshop on Urban Computing (UrbComp'12) 25-32 (2012).

50. Lessler, J. et al. Location-specific patterns of exposure to recent pre-pandemic strains of influenza A in southern China. Nat. Commun. 2, 423 (2011)

51. Crighton, E. J., Elliott, S. J., Moineddin, R., Kanaroglou, P. \& Upshur, R. E. An exploratory spatial analysis of pneumonia and influenza hospitalizations in Ontario by age and gender. Epidemiol. Infect. 135, 253-261 (2007).

52. Keeling, M. J. \& Rohani, P. Estimating spatial coupling in epidemiological systems: a mechanistic approach. Ecol. Lett. 5, 20-29 (2002).

53. Perc, M. Sustainable institutionalized punishment requires elimination of secondorder free-riders. Sci. Rep. 2, 344 (2012).

54. Szolnoki, A., Perc, M. \& Szabó, G. Defense mechanisms of empathetic players in the spatial ultimatum game. Phys. Rev. Lett. 109, 078701 (2012).

55. Gracia-Lazaro, C., Cuesta, J. A., Sánchez, A. \& Moreno, Y. Human behavior in Prisoner's Dilemma experiments suppresses network reciprocity. Sci. Rep. 2, 325 (2012).

56. Wilson, T. D., Houston, C. E., Etling, K. M. \& Brekke, N. A new look at anchoring effects: Basic anchoring and its antecedents. J. Exp. Psychol. 125, 387-402 (1996).

57. Furnham, A. \& Boo, H. C. A literature review of the anchoring effect. Journal of Socio-Economics 40, 35-42 (2011)

58. Heffernan, J. M., Smith, R. J. \& Wahl, L. M. Perspectives on the basic reproductive ratio. J. R. Soc. Interface 2, 281-293 (2005)

\section{Acknowledgments}

This work was partly supported by National Key Basic Research and Development Program (No.2010CB731403), the National Natural Science Foundation(No. 61273223), and the Research Fund for the Doctoral Program of Higher Education (No. 20120071110029) of China. LW also acknowledges the partial support by Fudan University Excellent Doctoral Research Program (985 Project).

\section{Author contributions}

L.W., Z.W. and X.L. planned the study; L.W., Y.Z. and Z.W. performed the experiments, analyzed the data, and prepared the figures; L.W., Z.W. and X.L. wrote the paper.

\section{Additional information} scientificreports 
Competing financial interests: The authors declare no competing financial interests. License: This work is licensed under a Creative Commons

Attribution-NonCommercial-NoDerivs 3.0 Unported License. To view a copy of this license, visit http://creativecommons.org/licenses/by-nc-nd/3.0/
How to cite this article: Wang, L., Wang, Z., Zhang, Y. \& Li, X. How human location-specific contact patterns impact spatial transmission between populations? Sci. Rep. 3, 1468; DOI:10.1038/srep01468 (2013) 